\title{
Scaling theory of DNA confined in nanochannels and nanoslits
}

\author{
Theo Odijk* \\ Complex Fluids Theory, Faculty of Applied Sciences, Delft University of Technology, 2628 BC Delft, The Netherlands
}

(Received 20 February 2008; published 9 June 2008)

\begin{abstract}
A scaling analysis is presented of the statistics of long DNA confined in nanochannels and nanoslits. It is argued that there are several regimes in between the de Gennes and Odijk limits introduced long ago. The DNA chain folds back on itself giving rise to a global persistence length that may be very large owing to entropic deflection. Moreover, there is an orientational excluded-volume effect between the DNA segments imposed solely by the nanoconfinement. These two effects cause the chain statistics to be intricate leading to nontrivial power laws for the chain extension in the intermediate regimes. It is stressed that DNA confinement within nanochannels differs from that in nanoslits because the respective orientational excluded-volume effects are not the same.
\end{abstract}

DOI: 10.1103/PhysRevE.77.060901

PACS number(s): 87.80.Fe, 81.05.Lg, 61.25.he, 61.30.Hn

A perusal of the rapidly developing literature on nanoconfined DNA shows that its behavior is more complex than anticipated (see, e.g., [1-17] ). It appears that more regimes are needed besides those originally described by Daoud and de Gennes [18] and Odijk [19]. The nanoconfinement of a semiflexible chain specifically introduces subtleties in the chain statistics that I address here within a scaling analysis. A complete theory would involve solving a Fokker-Planck equation subject to the boundary conditions arising from nanoconfinement [20]. Nevertheless, backfolding or hairpin formation may be addressed in a mechanical approximation [21], though entropic depletion of the chain near a wall still needs to be resolved quantitatively [22]. Numerical investigations of nanoconfined stiff chains interacting via excludedvolume interactions have appeared recently [23-25] but in the limit of ground-state dominance without accounting for hairpin formation.

Let us first consider a very long double-stranded DNA chain confined in a nanochannel of square cross section whose side $D$ is smaller than the persistence length $P$ so that we are in the Odijk regime $(D<P)$. Thus the chain may be conveniently viewed as a sequence of deflection segments of typical length [19]

$$
\lambda \simeq D^{2 / 3} P^{1 / 3} .
$$

The orientational fluctuations with respect to the channel center axis are given by the mean-square average

$$
\left\langle\theta^{2}\right\rangle \simeq c_{1}\left(\frac{D}{P}\right)^{2 / 3} .
$$

It is important to note that the coefficient $c_{1}$ here is quite small, as has been determined numerically [26-28] and estimated analytically [12]. Inevitably, a long chain must bear thermally activated hairpins leading to a global persistence length $g$ as shown in Fig. 1. The entropic depletion caused by the nanowalls forces the hairpin bends to be tightened up so that $g$ is often considerably larger than the persistence length $P$ [21]. Expressions for $g$ are presented in Appendix A.

\footnotetext{
*odijktcf@wanadoo.nl
}

In view of the backfolding, segments of the DNA interact with each other via the excluded-volume effect. Owing to the charges borne by the DNA backbone, one introduces an effective diameter $d_{\text {eff }}$ rather than a bare diameter [29] ( $P$ $\left.\gg d_{\text {eff }}\right)$. If the interaction were purely isotropic, the excluded volume between a pair of deflection segments would scale as [30]

$$
\beta_{\lambda} \simeq \lambda^{2} d_{\mathrm{eff}}
$$

But the segments are aligned [see Eq. (2)] so that the effective excluded volume becomes [31-33]

$$
\begin{gathered}
E=\beta_{\lambda}\langle|\sin \delta|\rangle, \\
\langle|\sin \delta|\rangle \simeq\left(\frac{D}{P}\right)^{1 / 3},
\end{gathered}
$$

where $\delta$ is the angle between two deflection rodlets (for a computation of the orientational factor, see Appendix B). It is stressed that we are in the sparse limit $d_{\text {eff }} \ll D$ : the orientational order is imposed solely by the walls of the nanochannel and independent of the density of DNA segments.

The DNA chain of length $L$ may now be viewed as a one-dimensional walk consisting of $L / g$ statistical segments. It is partly self-avoiding in the sense that the volume exclusion between the deflection rodlets is three-dimensional. Employing a mean-field argument of the Flory type that is excellent in one dimension [34], I write the free energy of the confined chain as

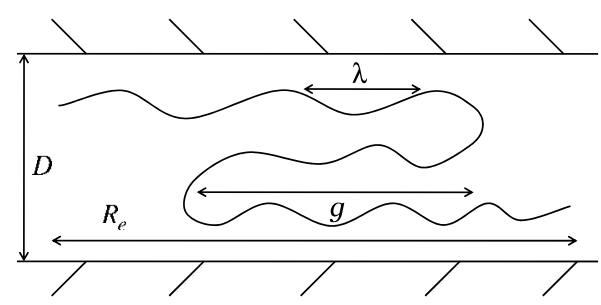

FIG. 1. A DNA chain enclosed in a nanochannel of width $D$. The distance between hairpins is typically $g$. 


$$
\frac{F}{k_{B} T} \simeq \frac{R_{e}^{2}}{L g}+\frac{N_{\lambda}^{2} E}{R_{e} D^{2}} .
$$

Here, $T$ is the temperature and $k_{B}$ is Boltzmann's constant. The first term in Eq. (6) is the ideal free energy needed to extend the DNA chain to root-mean-square extension $R_{e}$ and the second term arises from the interaction of $N_{\lambda}=L / \lambda$ deflection segments in a volume $R_{e} D^{2}$. Minimization of $F$ with respect to $R_{e}$ yields

$$
\begin{gathered}
R_{e} \simeq L \xi_{1}^{1 / 3}, \\
\xi_{1} \equiv \frac{g E}{\lambda^{2} D^{2}} \simeq \frac{g d_{\mathrm{eff}}}{D^{5 / 3} P^{1 / 3}} .
\end{gathered}
$$

Equations (6) and (7) are reminiscent of those occurring in the theory of grafted polymers $[35,36]$. One is now naturally led to introduce the following regimes.

Regime 1: $\xi_{1}>1$. From Eq. (7), we discern that the chain must be almost fully aligned $\left(R_{e} \simeq L\right)$ apart from minor fluctuations given by Eq. (2).

Regime $2 A: \xi_{1}<1$ provided $L>L_{*}$. The excluded-volume term in Eq. (6) is analogous to the excluded-volume parameter $Z$ introduced in the two-parameter theory of the expansion of flexible polymer chains [37],

$$
Z \simeq \frac{N_{\lambda}^{2} E}{D^{2} R_{e}} .
$$

The excluded-volume effect is fully exerted in the limit $Z$ $\gg 1$. There is a crossover to the case of weakly interacting segments at $Z=O(1)$. Hence, the contour length must be larger than $L_{*}$ if Eq. (7) is to remain valid.

$$
L_{*}=g \xi_{1}^{2 / 3} \simeq g^{1 / 3} D^{10 / 9} P^{2 / 9} d_{\mathrm{eff}}^{-2 / 3} .
$$

Regime $2 B: L<L_{*}$. In this case, the excluded-volume effect is weak $(Z \ll 1)$ so the behavior of the DNA is effectively that of an ideal chain as long as $L \gg g$,

$$
R_{e}^{2} \simeq L g
$$

I now investigate what happens as the nanochannel is widened. The global persistence length rapidly approaches the usual persistence length [see Eq. (A2) in Appendix A]. Accordingly, one may introduce a crossover

$$
D_{*}=c_{2} P
$$

at $g \simeq P$, which signals the breakdown of the Odijk regime (the numerical coefficient $c_{2}$ is larger than unity). Concomitantly, the channel is no longer narrow enough to impose orientational order on the DNA: $\langle|\sin \delta|\rangle=O(1)$ and $E$ $\simeq P^{2} d_{\text {eff. }}$. Thus, we now enter the next regime upon increasing $D$.

Regime 3: $D_{* *}>D>D_{*}$. I now express the total free energy of the chain as in Eq. (6) but with $g=P$. This leads to

$$
R_{e} \simeq L \xi_{2}^{1 / 3}
$$

$$
\xi_{2} \equiv \frac{P d_{\mathrm{eff}}}{D^{2}}
$$

It is again possible to demarcate one subregime in which the chain expansion is dominated by the excluded-volume effect from another subregime where the chain is ideal more or less. The crossover in the contour length is given by

$$
L_{*} \simeq \frac{P^{1 / 3} D^{4 / 3}}{d_{\mathrm{eff}}^{2 / 3}} .
$$

Superficially, it may appear as if Eq. (13) conforms to a Daoud-de Gennes type of theory [18], but this is not the case for $D<D_{* *}[$ see Eq. (16)]. The intermediate regime one has to introduce here is caused by the fact that the DNA segments are slender $\left(d_{\text {eff }} \ll P\right)$. The chain may be viewed as a sequence of anisometric blobs, each of length $\left(L_{*} P\right)^{1 / 2}$ and diameter $D$.

Regime 4: $D>D_{* *}$. Daoud and de Gennes argued that a flexible polymer confined in a capillary piles up as a sequence of blobs, each blob being viewed as a Flory chain of $m$ segments [18]. The blobs do not interpenetrate owing to the excluded-volume repulsion. The supposition is that $Z$ $\gg 1$ within a blob. In the problem at hand, we have $m$ segments of length $P$ interacting by an excluded volume $P^{2} d_{\text {eff }}$ yielding an excluded-volume parameter $Z=m^{2} \beta / m^{3 / 2} P^{3}$ $=m^{1 / 2}\left(d_{\mathrm{eff}} / P\right)$. If the Flory expansion is to be valid within a blob of radius $D$, we require $D \simeq\left(m^{1 / 2} P\right) Z^{1 / 5}$. In other words, upon eliminating $m$ we must have $Z=\left(D d_{\mathrm{eff}} / P^{2}\right)^{5 / 6}$. Therefore, if the blob picture is to be valid, one has to impose $Z$ $>1$ implying that $D_{* *}$ is expressed by

$$
D_{* *} \equiv P^{2} / d_{e f f} .
$$

Note that Eq. (13) remains valid as can be verified in a blob analysis. However, the difference between regimes 3 and 4 may show up in subtle measurements.

Let us next turn to nanoslits of rectangular cross section $A \times D(A>D)$. In many respects, the reasoning is now the same as that presented above so the analysis will be brief. I first focus on thin slits $(D \leq \pi P)$ in which the DNA chain is effectively one-dimensional.

The analogue of Eq. (6) is now

$$
\frac{F}{k_{B} T} \simeq \frac{R_{e}^{2}}{g L}+\frac{N_{\lambda}^{2} E}{R_{e} A D} .
$$

The orientational factor $\langle|\sin \delta|\rangle$ within $E$ then has a rather subtle dependence on $A$ and $D$ (see Appendix B). The deflection length is still given by Eq. (1). Upon minimizing Eq. (17), we get

$$
\begin{gathered}
R_{e} \simeq L \xi_{3}^{1 / 3}, \\
\xi_{3} \equiv \frac{g E}{A D} \simeq \frac{g d_{\mathrm{eff}}}{A^{2 / 3} D P^{1 / 3}} .
\end{gathered}
$$

The second equality in the expression for $\xi_{3}$ pertains to the limit $A \gg D$. It is again possible to introduce crossovers at $\xi_{3} \simeq 1$ and $L=L_{*}$ and so forth. 
If one next increases the width $A$, the global persistence length given by Eqs. (A1) and (A4) decreases rapidly to the value $P$ at $A=c_{3} P$, where $c_{3}$ is a numerical constant larger than unity. At the same time, the chain loses its local anisotropy: $\langle|\sin \delta|\rangle=O(1)$ [see Eq. (B3)]. It is important to realize that the chain remains confined to a thin slab $(D \lesssim \pi P)$. Equation (17) with $g=P$ still holds, though $\xi_{3}$ in Eq. (18) is replaced by

$$
\xi_{4} \equiv \frac{P d_{\mathrm{eff}}}{A D}
$$

Ultimately, if we keep on increasing $A$, we attain the case in which the chain may be viewed as a two-dimensional pancake $\left(A>R_{e}\right)$. Instead of Eq. (17), we have

$$
\frac{F}{k_{B} T} \simeq \frac{R_{e}^{2}}{P L}+\frac{N_{\lambda}^{2} E}{R_{e}^{2} D} .
$$

Minimization of $F$ with respect to $R_{e}$ yields

$$
R_{e} \simeq(L P)^{1 / 2}\left(\frac{L d_{\mathrm{eff}}}{P D}\right)^{1 / 4}
$$

One recognizes the usual $\frac{3}{4}$ power law applicable to the excluded-volume effect in two dimensions [36]. The excluded-volume parameter

$$
Z \simeq \frac{L d_{\mathrm{eff}}}{P D}
$$

has to be greater than unity if Eq. (23) is to be valid, otherwise $R \simeq(L P)^{1 / 2}$.

The analysis given above has the drawback in that the numerical coefficients are unknown and may deviate substantially from unity. This is exemplified in the application of Eqs. (8) and (10) to the recent measurements on $\lambda$-phage DNA extended within nanochannels of essentially square cross sections [3]. For instance, in the widest channel of width $D=440 \mathrm{~nm}$, the dimensionless parameter $\xi_{1}$ is about 0.11 and $g=2.3 \mu \mathrm{m}$, which would lead to a crossover length $L_{*}$ of about $10 \mu \mathrm{m}$ (I have estimated $d_{\text {eff }}$ to be $4.6 \mathrm{~nm}$ on the basis of the concentration of buffer used by Reisner et al.). But we know that the coefficients in Eqs. (2) and (3) are somewhat smaller than unity (see also Ref. [30]). In addition, there is a persistent finite-size effect for semiflexible chains that significantly suppresses the excluded-volume interaction $[38,39]$. On the whole, $L_{*}$ could be an order of magnitude larger so that the DNA of contour length $L=63 \mu \mathrm{m}$ would only be slightly perturbed by excluded volume. This would explain why the hairpin theory for the phantom worm [21] agrees well with the DNA extensions [3]. In a similar vein, Krishnan and Petrov [17] use the same theory to explain the ionic-strength dependence of the DNA elongation measured in some detail by Reisner et al. [13]. Theoretically, the strong dependence on salt then arises from the exponential dependence of $g$ on the persistence length $P$ [see Eq. (A2)] [17]. The latter quantity was dealt with on an empirical level by using the values from optical tweezer experiments [40]. However, Reisner et al. [13] themselves argue that a blob picture could be valid on the basis of assuming $g \equiv P$ (this would be regime 3 defined above). A reassessment of these experiments is warranted to reconcile the apparently opposing points of view.

As I argued above, the degree of orientational order depends on whether the cross section of the channel in which the DNA is confined is square, rectangular, or slitlike of infinite extent. In the case of the $1000 \times 100 \mathrm{~nm}^{2}$ nanoslits used in Ref. [12], the quantity $\xi_{3}=44$ from Eq. (19) turns out to be very large because the ionic strength is quite low $\left(d_{\text {eff }}=79 \mathrm{~nm}\right)$. The DNA is predicted to be fully extended even in $100-\mu \mathrm{m}$-long slits as is indeed the case. Bonthuis et al. [16] have studied the radius of gyration of DNA in twodimensional slits as a function of height $D$ right into the Odijk regime. There is only one abrupt transition at $D \simeq 2 P$, i.e., there are no intermediate regimes in agreement with the analysis presented here.

In summary, a scaling analysis of nanoconfined DNA has been presented based on orientational order imposed by the channel walls and a global persistence length greatly enhanced by entropic depletion. Clearly, more experiments are needed to delineate the regimes proposed here.

I thank Peter Prinsen for logistic help and David C. Schwartz, Madhavi Krishnan, Walter Reisner, Douwe Jan Bonthuis, and Christine Meyer for discussions and correspondence.

\section{Appendix A}

The global persistence length has been computed in the mechanical limit for the hairpin configurations (see [21]; the bent DNA remains double-stranded),

$$
g=3.3082 \bar{r} \exp [F(\bar{r})] / k_{B} T .
$$

In the case of nanochannels of square cross section, the free energy of a hairpin bend is given by

$$
F_{s}(\bar{r}) / k_{B} T=\frac{E_{m} P}{\bar{r}}-3 \ln \left(\frac{D-\bar{r} \sqrt{2}}{D}\right)-\ln \left(\frac{8}{3 \pi}\right)
$$

with an optimum radius

$$
\bar{r}=\frac{1}{6}\left[\left(E_{m}^{2} P^{2}+6 \sqrt{2} E_{m} D P\right)^{1 / 2}-E_{m} P\right]
$$

$\left(E_{m}=1.5071\right)$. In the case of nanoslits, these variables are

$$
\begin{gathered}
F_{\text {slit }}(\bar{r})=\frac{E_{m} P}{\bar{r}}-\ln \left[\left(\frac{A-2 \bar{r}}{A}\right) \frac{D}{\pi \bar{r}}\right]+1, \\
\bar{r}=\frac{E_{m} P A}{A+2 E_{m} P} .
\end{gathered}
$$

As $A$ becomes very large, $g$ tends to $P$, at least to the leading order. The limit is not precise because ultimately fluctuations cause the mechanical approximation to break down [21].

\section{Appendix B}

To a good approximation, the orientation-translation distribution of the long DNA is that of a chain confined in an 


\section{THEO ODIJK}

appropriate harmonic well [12]. Integrating over the translational degrees of freedom, one is left with a Gaussian distribution,

$$
f \sim \exp \left(-\frac{1}{2} G_{D} \theta_{x}^{2}\right) \exp \left(-\frac{1}{2} G_{A} \theta_{y}^{2}\right)
$$

for a fluctuating DNA segment [the nanoslit is $D$ wide in the $x$ direction and $A$ wide in the $y$ direction; $G_{D} \simeq(P / D)^{2 / 3}$ and $G_{A} \simeq(P / A)^{2 / 3}$; see Eq. (2)]. Hence, we have
PHYSICAL REVIEW E 77, 060901(R) (2008)

$$
\begin{aligned}
\langle|\sin \delta|\rangle= & \int_{-(1 / 2) \pi}^{(1 / 2) \pi} d \theta_{x} \int_{-(1 / 2) \pi}^{(1 / 2) \pi} d \theta_{y} \int_{-(1 / 2) \pi}^{(1 / 2) \pi} d w_{x} \\
& \times \int_{-(1 / 2) \pi}^{(1 / 2) \pi} d w_{y} f\left(\theta_{x}, \theta_{y}\right) f\left(w_{x}, w_{y}\right)|\sin \delta|
\end{aligned}
$$

[the appropriate normalization is included in Eq. (B2)]. To the leading order, integrals like Eq. (B2) are readily estimated $[31,41]$,

$$
\langle|\sin \delta|\rangle \simeq\left[\frac{G_{D}+G_{A}+1}{\left(G_{D}+1\right)\left(G_{A}+1\right)}\right]^{1 / 2},
$$

where the constant unity has been added to ensure that $\langle|\sin \delta|\rangle=O(1)$ in the isotropic limit.
[1] J. O. Tegenfeldt, C. Prinz, H. Cao, S. Chou, W. W. Reisner, R. Riehn, Y. M. Wang, E. C. Cox, J. C. Sturm, P. Silberzan, and R. H. Austin, Proc. Natl. Acad. Sci. U.S.A. 101, 10979 (2004).

[2] C. H. Reccius, J. T. Mannion, J. D. Cross, and H. G. Craighead, Phys. Rev. Lett. 95, 268101 (2005).

[3] W. Reisner, K. J. Morton, R. Riehn, Y. M. Wang, Z. Yu, M. Rosen, J. C. Sturm, S. Y. Chou, E. Frey, and R. H. Austin, Phys. Rev. Lett. 94, 196101 (2005).

[4] R. Fan, R. Karnik, M. Yue, D. Li, A. Majumdar, and P. Yang, Nano Lett. 5, 1633 (2005).

[5] J. T. Mannion, C. H. Reccius, J. D. Cross, and H. G. Craighead, Biophys. J. 90, 4538 (2006).

[6] A. Balducci, P. Mao, J. Han, and P. S. Doyle, Macromolecules 30, 6273 (2006).

[7] D. Stein, F. H. J. van der Heyden, W. J. A. Koopmans, and C. Dekker, Proc. Natl. Acad. Sci. U.S.A. 103, 15853 (2006).

[8] J. H. Kim, Y. R. Dukkipati, S. W. Pang, and R. G. Larson, Nanoscale Res. Lett. 2, 185 (2007).

[9] C. C. Hsieh, A. Balducci, and P. S. Doyle, Macromolecules 40, 5196 (2007).

[10] J. Tang and P. S. Doyle, Appl. Phys. Lett. 90, 224103 (2007).

[11] A. Balducci, C. C. Hsieh, and P. S. Doyle, Phys. Rev. Lett. 99, 238102 (2007).

[12] K. Jo, D. M. Dhingra, T. Odijk, J. J. de Pablo, M. D. Graham, R. Runnheim, D. Forrest, and D. C. Schwartz, Proc. Natl. Acad. Sci. U.S.A. 104, 2673 (2007).

[13] W. Reisner, J. P. Beech, N. B. Larsen, H. Flyvbjerg, A. Kristensen, and J. O. Tegenfeldt, Phys. Rev. Lett. 99, 058302 (2007).

[14] M. Krishnan, I. Mönch, and P. Schwille, Nano Lett. 7, 1270 (2007).

[15] J. D. Cross, E. A. Strychalski, and H. G. Craighead, J. Appl. Phys. 102, 024701 (2007).

[16] D. J. Bonthuis, C. Meyer, D. Stein, and C. Dekker (unpublished).

[17] M. Krishnan and E. P. Petrov, e-print arXiv:cond-mat/ 0805210 .
[18] M. Daoud and P. G. de Gennes, J. Phys. (Paris) 38, 85 (1977)

[19] T. Odijk, Macromolecules 16, 1340 (1983).

[20] A. N. Semenov, Eur. Phys. J. E 9, 353 (2002).

[21] T. Odijk, J. Chem. Phys. 125, 204904 (2006).

[22] T. Odijk, Macromolecules 20, 1423 (1987).

[23] J. Z. Y. Chen, D. E. Sullivan, and X. Yuan, Europhys. Lett. 72, 89 (2005)

[24] J. Z. Y. Chen and D. E. Sullivan, Macromolecules 39, 7769 (2006).

[25] J. Z. Y. Chen, D. E. Sullivan, and X. Yuan, Macromolecules 40, 1187 (2007)

[26] J. Wang and H. Gao, J. Chem. Phys. 123, 084906 (2005).

[27] Y. Yang, T. W. Burkhardt, and G. Gompper, Phys. Rev. E 76, 011804 (2007).

[28] F. Wagner, G. Lattanzi, and E. Frey, Phys. Rev. E 75, 050902(R) (2007).

[29] D. Stigter, Biopolymers 16, 1435 (1977).

[30] T. Odijk, Biopolymers 18, 3111 (1979).

[31] L. Onsager, Ann. N.Y. Acad. Sci. 51, 627 (1949).

[32] A. R. Khokhlov and A. N. Semenov, Physica A 108, 546 (1981).

[33] T. Odijk, Macromolecules 19, 2313 (1986).

[34] P. G. de Gennes, Scaling Concepts in Polymer Physics (Cornell University Press, Ithaca, NY, 1979).

[35] S. Alexander, J. Phys. (Paris) 38, 983 (1977).

[36] P. G. de Gennes, Macromolecules 13, 1069 (1980).

[37] H. Yamakawa, Modern Theory of Polymer Solutions (Harper and Row, New York, 1971).

[38] H. Yamakawa and W. H. Stockmayer, J. Chem. Phys. 57, 2843 (1972).

[39] J. Moon and H. Nakanishi, Phys. Rev. A 44, 6427 (1991).

[40] C. G. Baumann, S. B. Smith, V. A. Bloomfield, and C. Bustamante, Proc. Natl. Acad. Sci. U.S.A. 94, 6185 (1997).

[41] T. Odijk and H. N. W. Lekkerkerker, J. Phys. Chem. 89, 2090 (1985). 